\title{
Prejuízos da neuropatia periférica induzida por quimioterapia no cotidiano de pacientes com câncer de mama
}

\author{
Impairments of chemotherapy-induced peripheral neuropathy in the daily lives of patients \\ with breast cancer
Deficiencias de la neuropatía periférica inducida por quimioterapia en la vida diaria de pacientes con cáncer de mama

Márcio Alves Ribeiro ${ }^{1 *}$, Mary Elizabeth de Santana ${ }^{1}$, Amanda Rodrigues Figueiredo².

\begin{abstract}
RESUMO
Objetivo: Caracterizar os prejuízos impostos pela Neuropatia Periférica Induzida por Quimioterapia (NPIQ) na vida diária de pacientes com câncer de mama pós-tratamento quimioterápico. Métodos: Trata-se de um estudo descritivo, com abordagem quantitativa, com aplicação do instrumento validado Chemotherapy Induced Peripheral Neuropathy Assessment Tool (CIPNAT), abordando os prejuízos da NPIQ na vida das pacientes submetidas à quimioterapia. Participaram 196 pacientes com câncer de mama de um Centro de Tratamento Quimioterápico de Belém/PA. A análise baseou-se na estatística descritiva. Resultados: Em média, as atividades mais prejudicadas foram, no escore CIPNAT de 0 a 10: praticar atividade física $(6,98 \pm 0,93)$; aproveitar a vida $(6,78 \pm 1,35)$; realizar tarefas domésticas $(6,61 \pm 1,33)$ e trabalhar $(6,09 \pm 1,74)$. Conclusão: A NPIQ prejudica a vida das pacientes e ocasiona déficits nas atividades cotidianas, trabalho e campos sociais. Assim, a enfermagem deve atuar de modo integral, para aplicar um cuidar que contemple todos os domínios afetados; ademais, compreende-se que instrumento CIPNAT pode contribuir para aperfeiçoar a assistência de enfermagem prestada.
\end{abstract}

Palavras-chave: Quimioterapia, Doenças da junção neuromuscular, Neoplasias da mama, Enfermagem.

\begin{abstract}
Objective: To characterize the damage caused by Chemotherapy-Induced Peripheral Neuropathy (CIPN) in the daily life of patients with breast cancer after chemotherapy treatment. Methods: This is a descriptive study, with a quantitative approach, with the application of the validated Chemotherapy Induced Peripheral Neuropathy Assessment Tool (CIPNAT), addressing the damage of CIPN in the lives of patients undergoing chemotherapy. 196 breast cancer patients from a Chemotherapy Treatment Center in Belém/PA participated. The analysis was based on descriptive statistics. Results: On average, the most affected activities were, in the CIPNAT score from 0 to 10 : practicing physical activity $(6.98 \pm 0.93)$; enjoy life $(6.78 \pm 1.35)$; perform household chores $(6.61 \pm 1.33)$ and work (6.09 \pm 1.74$)$. Conclusion: NPIQ harms patients' lives and causes deficits in daily activities, work and social fields. Thus, nursing must act in an integral way, to apply care that includes all affected domains; furthermore, it is understood that the CIPNAT instrument can contribute to improving the nursing care provided.
\end{abstract}

Keywords: Chemotherapy, Neuromuscular junction diseases, Breast neoplasms, Nursing.

\section{RESUMEN}

Objetivo: Caracterizar el daño causado por la Neuropatía Periférico Inducida por Quimioterapia (NPIQ) en la vida diaria de pacientes con cáncer de mama después del tratamiento con quimioterapia. Métodos: Se trata de un estudio descriptivo, con abordaje cuantitativo, con la aplicación de la validada Herramienta de Evaluación de Neuropatía Periférica Inducida por Quimioterapia (HENPIQ), que aborda el daño del NPIQ en la vida de pacientes sometidos a quimioterapia. Participaron 196 pacientes con cáncer de mama de un Centro de Tratamiento de Quimioterapia en Belém/PA. El análisis se basó en estadística descriptiva. Resultados: En promedio, las actividades más afectadas fueron, en el puntaje HENPIQ de 0 a 10: práctica de actividad física (6,98 $\pm 0,93)$; disfrutar de la vida $(6,78 \pm 1,35)$; realizar las tareas del hogar $(6,61 \pm 1,33)$ y trabajar $(6,09 \pm 1,74)$. Conclusión: NPIQ daña la vida de los pacientes y provoca déficits en las actividades diarias, laborales y sociales. Así, la enfermería debe actuar de manera integral, para aplicar un cuidado que incluya todos los dominios afectados; además, se entiende que el instrumento HENPIQ puede contribuir a mejorar la atención de enfermería brindada.

Palabras clave: Quimioterapia, Enfermedades de la unión neuromuscular, Neoplasias mamarias, Enfermería.

${ }^{1}$ Universidade do Estado do Pará (UEPA), Belém - PA. *E-mail: marcioalves22@hotmail.com

${ }^{2}$ Centro Universitário Metropolitano da Amazônia (UNIFAMAZ), Belém - PA. 


\section{INTRODUÇÃO}

A neuropatia periférica é uma condição clínica caracterizada pelo comprometimento de nervos sensitivos e/ou motores periféricos, essencialmente nos membros e extremidades do corpo, que leva a alterações nas funções básicas de trânsito de informações entre o Sistema Nervoso Central (SNC) e a periferia do organismo, afetando diretamente as atividades típicas das estruturas nervosas. A etiologia da neuropatia periférica é múltipla, desencadeada em decorrência de patologias cujas manifestações incluem os distúrbios dos nervos periféricos. As principais doenças causadoras são a lesão por esforço repetitivo, a diabetes, o lúpus eritematoso sistêmico, o reumatismo, a psoríase, o hipotireoidismo, a hanseníase, casos de envenenamento por metais pesados e ainda, o efeito adverso do uso de determinados medicamentos, como certos antimicrobianos, estatinas e especialmente as drogas antineoplásica (HENRIQUE GCF, et al., 2019).

Especificamente quanto à quimioterapia para o câncer, um de seus principais efeitos adversos é a Neuropatia Periférica Induzida por Quimioterapia (NPIQ), caracterizada pela lesão progressiva nos tecidos nervosos periféricos (nervos e terminações nervosas), cujas fibras distais sofrem degradação funcional progressiva, especialmente do mecanismo de potencial em ação, decorrentes da exposição e depósito celular cumulativo de agentes neurotóxicos no transcurso dos ciclos de quimioterapia. As manifestações surgem em curtos a médios períodos, com variadas intensidades, conforme avança esse tratamento para 0 câncer (CURCIO KR, 2016).

A incidência da NPIQ varia entre $30 \%$ e $40 \%$ nos pacientes submetidos a múltiplos agentes antineoplásicos durante o ciclo de tratamento. $O$ efeito atinge em geral os membros, ascendendo de suas extremidades, é acompanhado de dor, dormência, formigamento e comprometimento das funções em níveis variados. A NPIQ está entre os aspectos que podem levar pacientes a não aderirem ao tratamento integralmente ou mesmo seu abandono, comprometendo sensivelmente as chances de cura e reabilitação. Ademais, a NPIQ está diretamente ligada ao declínio da capacidade para executar as tarefas do cotidiano, da autonomia e queda da qualidade de vida entre as pacientes (CAPONERO R, et al., 2016).

O câncer é uma doença que gera um quadro sindrômico complexo e, de seu próprio tratamento emanam efeitos indesejados, deletérios, de variadas complexidades, como sequelas físicas pós-cirúrgicas, efeitos adversos das drogas antineoplásicas, como a NPIQ; incluem-se também as manifestações psicológicas e a deterioração social relacionada à doença e ao seu tratamento; ocorrências diretamente ligadas aos sintomas que as pacientes experienciam (WAKIUCHI J, et al., 2019).

Por sua especificidade, é imprescindível se obter maior compreensão dos fatores relacionados à terapia antineoplásica e à NPIQ, para assim trazer maior clareza sobre seu prelúdio, desenvolvimento e fisiopatologia nos pacientes submetidos à quimioterapia e, ainda, favorecer, por meio do conhecimento, os atores da enfermagem, sejam pesquisadores e/ou assistentes, a aprimorarem técnicas, modelos assistenciais e de pesquisa para o cuidado prestado àqueles acometidos pela NPIQ durante a quimioterapia para o câncer. Isso possibilita abrandar o sofrimento imposto por esse efeito adverso, contribuindo para a qualidade de vida das pacientes e para o aperfeiçoamento do cuidado (MENDONÇA AB, et al., 2020).

Assim, ao se observar a notoriedade desse efeito adverso e na oportunidade de ampliar os conhecimentos sobre o tema, nesse estudo questionou-se: "Quais os prejuízos impostos pela neuropatia periférica induzida por quimioterapia na vida diária de pacientes com câncer de mama pós-tratamento quimioterápico?". E como objetivo central da pesquisa: Caracterizar os prejuízos impostos pela neuropatia periférica induzida por quimioterapia na vida diária de pacientes com câncer de mama pós-tratamento quimioterápico.

\section{MÉTODOS}

Tratou-se de um estudo descritivo, com abordagem quantitativa, desenvolvido através de uma pesquisa de campo. Esta ocorreu por meio da aplicação de uma escala ampla que denota os prejuízos advindos da $\mathrm{NPIQ}$ na vida das pacientes com câncer de mama, submetidas à quimioterapia e que desenvolvem esse efeito adverso. 
O instrumento aplicado foi o Chemotherapy Induced Peripheral Neuropathy Assessment Tool (CIPNAT), traduzido, adaptado culturalmente e validado para o Brasil em 2015 por Zandonai. Tal ferramenta adota escalas analógicas (de zero a dez pontos) para avaliar variados aspectos referentes ao cotidiano de convivência dos pacientes com a NPIQ, abrangendo temas como capacidade física, habilidades motoras, sono, conforto, dor, limitações para tarefas usuais e outros.

O escore permitiu avaliar o grau de interferência dos sinais e sintomas da NPIQ entre as participantes. O escore foi aplicado junto a Ficha de Coleta de Dados Sociodemográficos, um formulário próprio, concretizado após a leitura dos conteúdos de outras pesquisas do gênero e do modelo aplicado por Zandonai em seu estudo sobre a NPIQ (ZANDONAI AP, 2015).

A pesquisa ocorreu no Centro de Tratamento Quimioterápico de um hospital de referência em oncologia no estado do Pará. As participantes foram pacientes em pós-tratamento quimioterápico por câncer de mama, realizado com drogas de reconhecido potencial para desenvolver a NPIQ: taxanos (Paclitaxel e Docetaxel), alcaloides da vinca (Vincristina, Vimblastina e Vinorelbina) e agentes alquilantes (complexos da platina: Cisplatina, Carboplatina e Oxaliplatina), conforme os protocolos específicos aplicados durante as sessões quimioterápicas.

Os critérios de inclusão foram: sexo feminino; maiores de 18 anos de idade; com diagnóstico de câncer de mama como sítio primário; que finalizaram as sessões de tratamento por quimioterapia; aquelas que tiveram em seu protocolo de tratamento a utilização única ou associada dos grupos de fármacos: taxanos e/ou alcaloides da vinca e/ou complexos da platina e que estavam em acompanhamento ambulatorial pósquimioterápico.

Os critérios de exclusão foram: pacientes com afasia ou grande comprometimento da fala/comunicação ou cognição ou que aquelas que possuíam diagnóstico fechado de doença neuropática periférica prévia por outra causa alheia à quimioterapia para o câncer. A amostra foi probabilística do tipo aleatória simples, com 196 participantes, correspondendo a $49 \%$ da população feminina com câncer de mama em assistência na unidade.

A coleta das informações foi através de entrevista estruturada, individual em ambiente privado disponibilizado no setor. Na ocasião o questionário socioeconômico e a ficha de coleta do escore CIPNAT foram aplicados.

Os dados foram analisados a luz da estatística descritiva, com medidas de dispersão e de tendência central. A participação ocorreu estritamente após o esclarecimento, aceite e assinatura de Termo de Consentimento Livre e Esclarecido pelas participantes.

A pesquisa foi submetida ao Comitê de Ética e Pesquisa do Curso de Graduação em Enfermagem da Universidade do Estado do Pará, com parecer de aprovação no 2.819.075, de 14 de agosto de 2018.

\section{RESULTADOS}

A pesquisa obteve dados a partir de 196 participantes que atenderam aos critérios de inclusão e exclusão estabelecidos. As informações sociodemográficas demonstraram a predominância de faixa etária para adultas jovens, de 18 a 40 anos (25\%) e mulheres de meia idade, de 41 a 60 anos (54,59\%). A principal procedência das participantes da pesquisa foi a Região Metropolitana de Belém do Pará (64,80\%).

Quanto ao nível de escolaridade das participantes, se destacaram o ensino fundamental completo $(29,08 \%)$, seguido pelo ensino fundamental incompleto $(20,40 \%)$ e ensino médio completo $(19,89 \%)$. Dentre as atividades ou ocupações das participantes, a maior parcela é aposentada ou pensionista (26,02\%), ou exerce unicamente tarefas do lar (19,90\%), já as desempregadas representaram $9,20 \%$ do total. A faixa de renda familiar variou de um a dois salários mínimos (67,85\%).

A maior parte das participantes era casada ou mantendo relação estável $(52,04 \%)$ ou estava divorciada $(21,42 \%)$. A maternidade era predominante $(94,39 \%)$. O etilismo e tabagismo, eventual ou inveterado, teve ocorrência reduzida, de $10,71 \%$ e $16,84 \%$ respectivamente (Tabela 1). 
Tabela 1 - Frequência de informações sociodemográficas e hábitos de vida. $n=196.2018$.

\begin{tabular}{|c|c|c|}
\hline Variável & $\mathbf{N}$ & $\%$ \\
\hline \multicolumn{3}{|l|}{ Idade } \\
\hline $18-40$ & 49 & 25,00 \\
\hline $41-60$ & 107 & 54,59 \\
\hline$>60$ & 40 & 20,41 \\
\hline \multicolumn{3}{|l|}{ Procedência } \\
\hline Região metropolitana de Belém & 127 & 64,80 \\
\hline Capanema & 08 & 4,08 \\
\hline Cametá & 07 & 3,57 \\
\hline Abaetetuba & 07 & 3,57 \\
\hline Barcarena & 07 & 3,57 \\
\hline Braganca & 06 & 3,06 \\
\hline Outros municípios & 34 & 17,34 \\
\hline \multicolumn{3}{|l|}{ Escolaridade } \\
\hline Não alfabetizada & 08 & 4,08 \\
\hline Alfabetizada & 27 & 13,77 \\
\hline Fundamental completo & 57 & 29,08 \\
\hline Fundamental incompleto & 40 & 20,40 \\
\hline Ensino médio completo & 39 & 19,89 \\
\hline Ensino médio incompleto & 12 & 6,12 \\
\hline Superior / Pós-graduação & 13 & 6,63 \\
\hline \multicolumn{3}{|l|}{ Atividade/ocupação } \\
\hline Aposentada / pensionista & 51 & 26,02 \\
\hline Do lar & 39 & 19,90 \\
\hline Empregada doméstica & 16 & 8,16 \\
\hline Autônoma & 15 & 7,65 \\
\hline Comerciária & 13 & 6,63 \\
\hline Servidora pública & 13 & 6,63 \\
\hline Serviços de higienização & 08 & 4,08 \\
\hline Pescadora & 07 & 3,57 \\
\hline Professora & 06 & 3,06 \\
\hline Outras atividades & 10 & 5,10 \\
\hline Desempregada / sem ocupação & 18 & 9,20 \\
\hline \multicolumn{3}{|l|}{ Renda familiar (salários mínimos) } \\
\hline$<1$ & 14 & 7,14 \\
\hline 1 a 2 & 133 & 67,85 \\
\hline 3 a 4 & 37 & 18,88 \\
\hline$>4$ & 12 & 6,12 \\
\hline \multicolumn{3}{|l|}{ Estado civil } \\
\hline Solteira & 23 & 11,73 \\
\hline Casada / União estável & 102 & 52,04 \\
\hline Divorciada & 42 & 21,42 \\
\hline Viúva & 29 & 14,80 \\
\hline \multicolumn{3}{|l|}{ Maternidade } \\
\hline Sim & 185 & 94,39 \\
\hline Não & 11 & 5,61 \\
\hline \multicolumn{3}{|l|}{ Etilismo } \\
\hline Sim & 21 & 10,71 \\
\hline Não & 175 & 89,28 \\
\hline \multicolumn{3}{|l|}{ Tabagismo } \\
\hline Sim & 33 & 16,84 \\
\hline Não & 163 & 83,16 \\
\hline Total & 196 & 100 \\
\hline
\end{tabular}

Fonte: Ribeiro MA, et al., 2018. 
Ao investigar o quanto os sintomas da neuropatia periférica induzida por quimioterapia prejudicam a realização das atividades cotidianas, observou-se primeiramente que esse nível de interferência pode ser descrito, de acordo com o escore, como moderado abaixo, a depender do aspecto investigado. Destacaramse as seguintes atividades como as mais prejudicadas: exercitar-se/praticar atividade física; aproveitar a vida; realizar tarefas domésticas habituais e trabalhar. Todas as atividades exploradas são apresentadas com média e desvio padrão na tabela a seguir, cuja avaliação baseou-se na escala analógica de 0 a 10 , conforme o conteúdo do instrumento CIPNAT aplicado a cada participante (Tabela 2).

Tabela 2 - Nível de interferência relacionada às manifestações clínicas neuropáticas periféricas de pacientes submetidas à quimioterapia. n=196. 2018.

\begin{tabular}{lcc}
\hline $\begin{array}{c}\text { Interferência Relacionada aos } \\
\text { Sintomas da Neuropatia Periférica }\end{array}$ & $\begin{array}{c}\text { Média do Escore } \\
\text { (de 0 a 10) }\end{array}$ & $\begin{array}{c}\text { Desvio } \\
\text { Padrão }\end{array}$ \\
\hline Vestir & 4,58 & 1,14 \\
Andar & 2,85 & 0,81 \\
Pegar objetos & 3,93 & 0,76 \\
Segurar objetos & 3,47 & 1,43 \\
Dirigir* & 1,92 & 0,91 \\
Trabalhar & 6,09 & 1,74 \\
Participar de atividades de lazer & 5,59 & 0,85 \\
Exercitar/atividade física & 6,98 & 0,93 \\
Atividade sexual* & 4,40 & 1,04 \\
Dormir & 4,10 & 1,08 \\
Relacionamento com outras pessoas & 0,73 & 0,76 \\
Escrever & 4,15 & 0,77 \\
Realizar tarefas domésticas habituais & 6,61 & 1,33 \\
Aproveitar a vida & 6,78 & 1,35 \\
\hline
\end{tabular}

Legenda: *Itens com n inferior à totalidade (respectivamente 78 e 122 indivíduos), por haver participantes que referiram não realizar a atividade em questão.

Fonte: Ribeiro MA, et al., 2018.

\section{DISCUSSÃO}

Mediante os resultados obtidos, verificou-se que as faixas etárias predominantes incluíam as mulheres de meia idade e idosas. Culminando com o fato de que o surgimento do câncer de mama tem estreitamento com as diversas fases fisiológicas do corpo da mulher ao longo da vida, a fase da menopausa, as alterações hormonais, o tempo de exposição a fatores de risco ambientais e o próprio processo de envelhecimento. Os dados se alinham com outros estudos similares, como aquele conduzido no Ceará, que encontrou a faixa etária partindo de 50 anos como predominante (63,40\%) (TORRES DM, et al., 2016).

Quanto à demanda atendimento o predomínio é da região metropolitana e proximidades da capital, isso indica, especialmente para o Pará, um Estado de grande extensão, que além da maior concentração demográfica na área apontada, há a constante necessidade de expansão da rede de atenção ao câncer nas localidades mais distantes, sobretudo para meios diagnósticos precoces e tratamento avançado que ainda se concentram em Belém e em polos regionais, o que se traduz em amplas demandas de pacientes nesses centros, com tratamento fora de domicílio e dificuldades no acesso à rede, além da pressão sobre os serviços existentes (SALDANHA RF, et al., 2019).

Em relação ao grau de instrução, destacou-se entre as participantes o ensino fundamental completo ou incompleto, o que se alinha ao cenário atual da região norte do Brasil, que ainda figura com índice de $45,40 \%$ entre as mulheres (segundo mais baixo por regiões brasileiras) quanto à instrução formal e conclusão do ensino básico (BRASIL, 2020). Resultados similares foram observados em estudo sobre o perfil de pacientes com câncer de mama atendidas em um serviço de referência do Tocantins, no qual predominou o nível de escolaridade ensino fundamental incompleto, com $43,80 \%$ do total (SOUSA MM, et al., 2016). 
A renda das participantes era ligada sobretudo a aposentadorias, pensões e auxílios governamentais de programas de transferência direta de renda e auxílio doença. Como ocupação destacou-se o trabalho no lar ou doméstico, em alguns casos ligados à cultura local, à escolaridade ou à diminuição das capacidades na produção econômica em virtude da doença grave. Um estudo sobre o perfil epidemiológico de mulheres jovens com câncer de mama desenvolvido no nordeste apontou perfil semelhante, com $30 \%$ de atividade do lar e o destaque para pensões e auxílios previdenciários, que alcançavam entre um e dois salários mínimos, assim como neste estudo (SOUZA NHA, et al., 2017).

O perfil conjugal de casada prevaleceu, assim como a constituição de família, onde a maternidade foi quase que absoluta entre as mulheres pesquisadas. Esse perfil pode ser tanto de risco para o câncer de mama (no caso de início da atividade sexual precoce), quanto de proteção (amamentação é fator redutor de risco). Esses achados corroboram com aqueles de uma pesquisa desenvolvida em Pernambuco sobre adesão ao tratamento do câncer de mama, em que 52,2\% eram das participantes eram casadas (BUSHATSKY M, et al., 2018). Outro estudo, sobre perfil sociodemográfico de pacientes submetidas à mastectomia por câncer, em Minas Gerais, exibiu resultados semelhantes quanto à maternidade, 96,70\% possuíam prole (ALVARENGA JTA, et al., 2018).

Quanto ao consumo de álcool e tabaco, o destaque foi à baixa adesão a essa prática entre as pesquisadas, o que é bastante benéfico e favorece tanto o tratamento quanto o prognóstico das pacientes. Assim, esse achado permitiu observar que não houve forte relação entre surgimento do câncer e tabagismo e/ou etilismo para esse grupo. Um estudo de perfil clínico-epidemiológico de mulheres com câncer de mama em São Paulo obteve resultados similares, nele, apenas 0,3\% das participantes consumiam álcool, $14 \%$ eram tabagistas e 2,7\% ex-tabagistas. (MAGALHÃES G, et al., 2017).

Os resultados relativos à NPIQ permitiram caracterizar como esses efeitos adversos foram capazes de interferir de variadas formas na vida das pacientes em tratamento quimioterápico. O grau de interferência ou prejuízo na vida diária foi considerado, em geral, como moderado ou baixo em alguns aspectos avaliados. Observa-se que cada atividade cotidiana está relacionada a atribuições laborais, domésticas, mobilidade, locomoção, habilidades manuais, atividades sociais, interpessoais, de lazer, ou se ligam ao autocuidado; podendo sofrer desgastes em virtude dos efeitos da NPIQ durante e após os ciclos de quimioterapia (BAVIERA AF, et al., 2019).

Os pontos de maior desequilíbrio foram quanto à realização de atividade física regular, atividades domésticas habituais, trabalho; além de limitações para aproveitar a vida em geral e participação em atividades de lazer. Ressalta-se que essas conclusões das participantes também se relacionavam com o próprio câncer, considerado limitante e, associado aos sintomas adicionais gerados pelo NPIQ, se intensifica a sensação de desgaste e limitação, afetando especialmente as rotinas e o papel social e familiar das pacientes acometidas (CAVALER AW, et al., 2017).

A exacerbação dos sintomas neuropáticos se manifesta com o surgimento e sustentação de déficits especialmente concentrados nos membros e extremidades, também se associando à sensibilidade alterada e a dor. Esses danos atentam às capacidades ligadas ao cotidiano e ao autocuidado que, outrora de simples execução, como vestir, abotoar, escovar os dentes, apanhar e segurar objetos nas mãos, dar um laço nos cadarços, entre outras, agora têm sensível declínio, limitando as habilidades de execução (PONTE S e RÉCIO MT, 2017).

A neurotoxicidade periférica ligada à quimioterapia tem incidência variada e atrelada ao tipo de fármaco, dose e tempo de exposição. Assim, de igual forma, o grau e a espécie de injúria aos tecidos e às capacidades sensitivas e motoras, é variado, em geral acarretando parestesias de extremidades que podem afetar o equilíbrio e a marcha, e também ocasionar déficits motores finos, o que repercute nas tarefas cotidianas e na autonomia das pacientes. Junto aos sintomas neuromotores, as dores neuropáticas, os distúrbios da propriocepção e equilíbrio e a fraqueza podem contribuir para dificuldades na deambulação, especialmente idosas, bem como aumentar o risco de quedas (KAMEO SY, et al., 2016). 
O estudo evidenciou que a NPIQ gera um ônus à execução de tarefas domésticas simples, diretamente ligadas ao cuidado familiar, do lar e próprio. Desta forma, os sintomas da NPIQ estão ligados a repercussões desagradáveis e limitantes e afetam a capacidade para o preparo de alimentos; a realização da organização do lar; o manuseio de instrumentos perfurocortantes; limitação sobre a utilização da força muscular e até mesmo o sofrimento causado pela exposição a pequenas diferenças de temperatura, como por exemplo, o ato abrir a geladeira pode gerar uma sensibilidade térmica exacerbada (FERREIRA LC, 2018).

Os danos da NPIQ sobre o trabalho são apontados pelas participantes como importantes, pois essa limitação tem impacto direto sobre a perspectiva socioeconômica e financeira, sendo objeto de preocupação e desgaste adicionais para as pacientes que enfrentam tal experiência. A redução da capacidade produtiva, da autonomia para o trabalho e a larga possibilidade de afastamento laboral são ocorrências pertinentes aos quadros gerados pela doença de base (o câncer) e pelos efeitos adversos da quimioterapia, especialmente os sintomas neuropáticos que têm ampla variedade e capacidade de gerar condições limitantes acabam por extrapolar o problema de saúde da paciente (CESAR ESL, et al., 2017).

O prejuízo neuromotor e sensitivo, as dores, a fraqueza e tonturas podem levar a limitações para membros superiores e inferiores, capazes de afetar, por exemplo, as habilidades para dirigir veículos ou operar máquinas, utilizar ferramentas pesadas e de precisão, atarraxar, pregar, grampear, manusear artefatos para corte, costura, utilização de equipamentos ou máquinas variadas, escrever, digitar e tantas outras atividades em que as mulheres estão inseridas, sejam as de escritório, indústria, produção ou serviços. Pode ocorrer a dificuldade para o manuseio de materiais específicos a cada profissão e desta forma, fica claro que a NPIQ pode inabilitar para o trabalho de modo temporário ou permanente, sendo então necessário o suporte financeiro de terceiros ou do sistema previdenciário, e assim, acaba emergindo indiretamente um problema socioeconômico (SCISCI N, 2016).

Os sintomas manifestos da NPIQ também tendem a prejudicar o campo social, o lazer e o recreio, as relações interpessoais e, desta forma, podem subjugar a limitações nessas necessidades. As participantes sinalizaram aspectos como a interferências em atividades de lazer, na realização de atividades físicas regulares. Isso se deve especialmente a quadros como a fraqueza, as dores e alterações na sensibilidade térmica; capazes de limitar as participações em eventos que possam exigir esforço físico maior ou longa jornada, assim como, ambientes climatizados podem gerar desconforto adicional (LIMA CVC e SANTOS WM, 2017).

Nessa perspectiva além do próprio convívio com o câncer de mama, os sintomas clínicos da NPIQ podem condicionar a gregária apenas a eventos que compatibilizem a necessidade de bem-estar e conforto razoáveis para paciente com limitações físicas advindas da doença de base e seu tratamento. As relações familiares e sociais tendem a sofrer prejuízos, observados tanto pelas pacientes que vivem as experiências, compreendidos como limitantes; como pelos membros da rede familiar e social delas, que, por motivos diversos, limitação de informações ou receio, podem indiretamente não garantir ambiências agregadores e limitar a inserção e gregária, que são extremamente necessárias para as pacientes (SILVA SED, et al., 2018).

Deste modo, é notório que a avaliação clínica precoce e contínua é um importante meio para qualificar a assistência a ser prestada a essas pacientes e, para isso, utilizar instrumentos validados cientificamente é um contribuinte para esse intento. Ademais, a enfermagem atua rotineiramente prestando orientações sobre aos efeitos adversos da quimioterapia, incluindo a NPIQ, gerando vínculo, confiança e contribuindo para o estímulo ao autocuidado e manutenção da autonomia das pacientes. Resultados como os apresentados denotam a necessidade crescente da elaboração de planos de cuidados mais incisivos, especialmente protocolos institucionais que, além de estabelecer as formas de cuidar, também possuam metas/resultados assistenciais para pacientes com esse perfil e, assim, se estabeleçam terapêuticas multidisciplinares de maior eficiência (MORETTO IG, et al., 2019). 


\section{CONCLUSÃO}

A NPIQ é um efeito adverso ligado à quimioterapia antineoplásica que, embora não alcance níveis de mortalidade, causa prejuízos e limitações importantes que refletem na qualidade de vida. Por intermédio deste estudo pôde-se identificar a ocorrência da NPIQ; bem como caracterizar os prejuízos impostos pela $\mathrm{NPIQ}$ na vida diária de pacientes com câncer de mama pós-tratamento quimioterápico. Os sintomas da NPIQ prejudicam direta ou indiretamente a vida das pacientes e ocasionam déficits para o desempenho das atividades cotidianas, limitações nas relações sociais, familiares, no lazer e impactos no trabalho. Assim, a enfermagem e equipe multiprofissional devem atuar com uma visão holística que contemple todos os domínios afetados pelo efeito adverso. A pesquisa exibe resultados capazes de guiar as condutas clínicas e abre precedente a novas reflexões e abordagens para um melhor cuidar. Como limitação observa-se que a exploração qualitativa sobre os achados foi reduzida, a qual, sob outro olhar, também poderia enriquecer a análise.

\section{REFERÊNCIAS}

1. ALVARENGA JTA, et al. Perfil socioeconômico, demográfico e indicativo de depressão em mulheres submetidas à mastectomia no pós-operatório tardio. Revista de Enfermagem e Atenção a Saúde (online), 2018; 7(2): 3-16.

2. BAVIERA AF. Acupuntura em adultos com neuropatia periférica induzida por quimioterapia: uma revisão sistemática. Revista Latino-Americana de Enfermagem, 2019; 27: 1-10.

3. BUSHATSKY M, et al. Mulheres com câncer de mama: adesão com tamoxifeno. Ciência, Cuidado e Saúde, 2018; 17(3): 1-7.

4. BRASIL. Instituto Brasileiro de Geografia e Estatística. Pesquisa Nacional por Amostra de Domicílios Contínua (PNAD). 2020. Disponível em: https://biblioteca.ibge.gov.br/visualizacao/livros/liv101736_informativo.pdf. Acessado em: 19 de Novembro de 2020.

5. CAPONERO R, et al. Neuropatia pós-quimioterapia. Revista Dor, 2016; 17(suppl 1): 56-58.

6. CAVALER AW, et al. Assistência de enfermagem frente aos efeitos colaterais em pacientes submetidos a quimioterapia. RIES, 2017; 6(1): 200-212.

7. CESAR ESL, et al. Qualidade de vida de mulheres com câncer mamário submetidos à quimioterapia. Revista Rene, 2017; 18(5): 679-686.

8. CURCIO KR. Instruments for assessing chemotherapy- induced peripheral neuropathy: a review of the literature. Clinical Journal of Oncology Nursing, 2016; 20(2): A1-A8.

9. FERREIRA LC. Neuropatia periférica induzida por quimioterapia e sua associação com quedas e síndrome das pernas inquietas. Dissertação (Mestrado em Ciências da Saúde) - Universidade Federal de Sergipe, Aracaju, 2018; $80 \mathrm{p}$.

10. HENRIQUE GCF, et al. Intervenções não farmacológicas no tratamento da neuropatia periférica induzida pela quimioterapia. Revista Eletrônica Acervo Saúde, 2019; Vol.Sup.28: 1-8.

11. KAMEO SY, et al. Mucosite, neuropatia periférica e síndrome mão-pé: ocorrências e reflexões para o cuidado de enfermagem. Revista de Enfermagem UFPE on line, 2015; 9(9): 9246-9253.

12. LIMA CVC, SANTOS WM. Vivencias recorrentes de mulheres com câncer de mama durante a quimioterapia. Revista Saúde e Desenvolvimento, 2017; 11(9): 1-16.

13. MAGALHAES G, et al. Perfil clínico, sociodemográfico e epidemiológico da mulher com câncer de mama. Revista de Pesquisa Cuidado é Fundamental (online), 2017; 9(2): 473-479.

14. MENDONÇA $A B$, et al. Sofrimento de pacientes com câncer em quimioterapia neurotóxica: uma abordagem fenomenológica. Texto \& Contexto Enfermagem, 2020; v.29: 1-17.

15. MORETTO IG, et al. Acompanhamento por telefone como intervenção de enfermagem a pacientes em quimioterapia ambulatorial: revisão integrativa. Revista Gaúcha de Enfermagem, 2019; 40: 1-12.

16. PONTE S, RÉCIO MT. Neuropatia periférica induzida pela quimioterapia. On 34, 2017; 10(1): $26-29$.

17. SALDANHA RF, et al. Estudo de análise do fluxo de pacientes de câncer de mama no Brasil entre 2014 e 2016. Cadernos de Saúde Pública, 2019; 35(7): 1-14.

18. SCISCI N. prevalência e característica da dor neuropática e neuropatia periférica em doentes submetidos a oxaliplatina para tratamento do câncer colorretal. Dissertação (Mestrado em Ciências) - Faculdade de Medicina, Universidade de São Paulo, São Paulo, 2016; 169 p.

19. SILVA SED, et al. Os impactos da terapia quimioterápica e as implicações para manutenção do cuidado. Um estudo de representações sociais. Revista de Pesquisa Cuidado é Fundamental (Online), 2018; 10(2): 516-523.

20. SOUSA MM, et al. Perfil clínico-epidemiológico de mulheres com neoplasia de mama atendidas no hospital regional de referência no município de Araquaína-TO no período de 2000 a 2015. DESAFIOS: Revista Interdisciplinar da Universidade Federal de Tocantins, 2016; 2(2): 283-306.

21. SOUZA NHA, et al. Câncer de mama em mulheres jovens: estudo epidemiológico no nordeste brasileiro.

22. SANARE (Sobral. Online), 2017; 16(2): 60-67.

23. TORRES DM, et al. Análise de dados epidemiológicos de pacientes acompanhadas por neoplasia mamária em um hospital de Fortaleza (CE). Revista Brasileira de Mastologia, 2016; 26(2): 39-44.

24. WAKIUCHI J, et al. Reconstruindo a subjetividade a partir da experiência do câncer e seu tratamento. Revista Brasileira de Enfermagem, 2019; 72(1): 133-141.

25. ZANDONAI AP. Adaptação transcultural e validação do instrumento Chemotherapy- Induced Peripheral Neuropathy Assessment Tool (CIPNAT) para o Brasil. Tese (Doutorado em Ciências) - Escola de Enfermagem de Ribeirão Preto. Universidade de São Paulo, Ribeirão Preto, 2015; 199 p. 